\title{
ORTHOCENTRIC PROPERTIES OF THE PLANE DIRECTED $n$-LINE*
}

\author{
BY \\ JOSEPH ELLIS HODGSON
}

Introduction.

In a memoir entitled Orthocentric Properties of the Plane $n$-Line, $\dagger$ Professor MoRley has found for $n$ lines of a plane natural metrical analogues of the elementary theorems that the perpendiculars from the vertices of a triangle to the opposite sides meet in a point, and that the four such points, or orthocenters, of the four triangles of a 4-line are on a line. It was in fact proved that, corresponding to the extended meaning of the term orthocenter for a higher number of lines, there is an orthocentric point for an odd number of lines beginning with three, while for an even number, beginning with four, there is a line of orthocenters, or, as it may be called, a directrix.

It is proposed in the following paper to prove for the system of $n$ directed lines in a plane general theorems corresponding in a way to those given in the above memoir for lines without specified direction. Added to this is the more or less detailed discussion of certain questions closely related to the main subject.

The method of vector analysis employed in this paper is due to Professor MoRLEY. $\ddagger$ In this method conjugate, or circular, coördinates are used almost exclusively, and the conjugate of a complex number $x$ is denoted by $\bar{x}$. A complex number of absolute value equal to unity is generally denoted by $t$ or $T$ and is called a turn.

The standard equation of a line is here taken in the form

$$
t x+\bar{x} / t-2 r=0,
$$

in which $r$ is real and equal to the length of the perpendicular from the origin to the line, and in which the clinant, or inclination function, is $-1 / t^{2}$.

A line may be considered as described by a point moving in either of two opposite directions. The locus of the centers of circles tangent to two such intersecting lines is composed of the two bisectors of the angles at the point of intersection; but if definite directions are first assigned to the lines and if the point describing the tangent circles is to move at the point of tangency

* Presented to the Society, February 24, 1912.

† These Transactions, vol. 4 (1903), pp. 1-12.

† These Transactions, vol. 1 (1900), p. 97. 
in the same direction as that given to the lines, the locus consists of only one line. If we adopt the convention that, when the sense ascribed to the line is such as to tend to turn the plane in a positive direction, the distance from line to point shall be positive if the origin and point are on opposite sides of the line, but negative if on the same side, then the points of the locus are alone equidistant from the intersecting lines. Hence the locus may be called the equidistant axis of the two directed lines. Three directed lines have three equidistant axes which meet in a point, the one point equidistant from the three lines.*

In the equation of the line

$$
t x+\bar{x} / t-2 r=0,
$$

$2 r$ is the distance from the origin to the reflection of the origin in the line. A line parallel to the given line and on the point $x_{0}$ is

$$
t x+\bar{x} / t-t x_{0}-\bar{x}_{0} / t=0 .
$$

The distance from the origin to the reflection of the origin in this line is $t x_{0}+\bar{x}_{0} / t$. The distance from one reflection to the other is, consequently,

$$
t x_{0}+\bar{x}_{0} / t-2 r \text {. }
$$

But this distance is twice the distance from the line to the point $x_{0}$. Hence the distance from line to point may be written

$$
\pm \frac{1}{2}\left(t x_{0}+\bar{x}_{0} / t-2 r\right) \text {. }
$$

The equations of two lines to which directions have been given are

$$
t_{1} x+x / t_{1}-2 r_{1}=0, \quad t_{2} x+\bar{x} / t_{2}-2 r_{2}=0 .
$$

Hence, remembering the convention as to signs, the equidistant axis of these two lines is

$$
\left(t_{1} x+\bar{x} / t_{1}-2 r_{1}\right)-\left(t_{2} x+\bar{x} / t_{2}-2 r_{2}\right)=0 .
$$

If now we reverse the direction of one of the lines the equidistant axis is the other of the pair of bisectors of the angles at the intersection of the given lines.

§1. Second circle for a directed 4-line. Directrix for a directed 5-line.

The so-called line equation in conjugate coördinates of the hypocycloid of class 4 , or astroid, is

$$
t^{4}-t^{3} x-t \bar{x}+1=0 .
$$

Another curve of the same type is one that has been designated the parastroid whose line equation in the same coördinates is

$$
t^{4}-t^{3} x+\mu t^{2}-t \bar{x}+1=0,
$$

* Loud, these Transactions, vol. 1 (1900), p. 323. 
where $\mu$ is real, since the equation must be self-conjugate. The latter curve is parallel to the former inasmuch as the distance from the origin to any line of the latter is

$$
\frac{t^{4}+\mu t^{2}+1}{2 \sqrt{t^{4}}}=\frac{1}{2}\left(t^{2}+1 / t^{2}+\mu\right) .
$$

The general parastroid is, therefore, parallel to that particular one for which $\mu=0$, that is, to the astroid. It is convenient to refer to the parastroid by the notation $A^{4}$.

Similarly, the curve

$$
t^{6}-\sigma_{1} t^{5}+t^{4} x-\mu^{\prime} t^{3}+t^{2} \bar{x}-\sigma_{5} t+1=0,
$$

of the same general type as the parastroid, may be designated the $A^{6}$. The $\sigma$ 's refer to the symmetric functions of the six roots of the equation while $\mu^{\prime}$ is real. Also, the $A^{6}$ is parallel to the curve

$$
t^{6}-\sigma_{1} t^{5}+t^{4} x+t^{2} \bar{x}-\sigma_{5} t+1=0 .
$$

There are of course similar curves of higher order corresponding to equations beginning with $t^{8}, t^{10}$, etc., and these may be briefly designated by the symbols $A^{8}, A^{10}$, etc. Again, the circle may be included in this type of curves and has as its general line equation in conjugate coördinates

in which $\rho$ is real.

$$
t^{2}(x-a)+(\bar{x}-\bar{a})-2 \rho t=0,
$$

It is to be noted that the clinant of each of the lines just given is $-1 / t^{2}$, the same therefore as that of the standard equation of a line used in this paper. The line of the astroid for instance is

and if

$$
t x+\frac{\bar{x}}{t}=t^{2}+\frac{1}{t^{2}}
$$

$$
t x+\frac{\bar{x}}{t}=2 r
$$

be a line to which a direction is assigned, the $r$ of the latter is the $\frac{1}{2}\left(t^{2}+1 / t^{2}\right)$ of the former. Moreover $t$ or $-t$ gives the same clinant in each. In form, then, the lines of the $A$ curves and the standard line are essentially the same, but the former are not conveniently employed where a reversal of direction is concerned-a question to be taken up later on. In what immediately follows, however, the use of the above type of curves as defined by their line equation affords a neat method of proving certain theorems relating to sets of 4 and 5 directed lines, and, in the next section of this paper, concerning $n$ directed lines in general. 
In the first place, then, consider the equation of the $A^{4}$

$$
t^{4}-x t^{3}+\mu t^{2}-\bar{x} t+1=0
$$

and the general line equation of the circle

$$
t^{2}(x-a)+(\bar{x}-\bar{a})-2 \rho t=0 .
$$

The elimination of $x$ and $\bar{x}$ gives

$$
t^{4}-a t^{3}+(\mu-2 \rho) t^{2}-\bar{a} t+1=0 .
$$

It is evident that this combination of the two equations selects from the eight common lines of the two curves a set of four, and with each of these lines of the set of four may be associated a direction, viz., the direction obtained by putting for $t_{i}$ in $-1 / t_{i}^{2}(i=1,2,3,4)$ one of the roots of the quartic in $t$ obtained on eliminating $x$ and $\bar{x}$ from (1) and (2).

In (3) $a=S_{1},(\mu-2 \rho)=S_{2}, \bar{a}=S_{3}$, and $1=S_{4}$, the $S$ 's being symmetric functions of the four roots of (3). It is to be observed that $S_{4}=1$ characterizes the four lines picked out of the eight common lines of the curves by combining their equations as above.

Since in (2) $a$ is the center of the circle, the general circle of the plane, and since in (3) $a=S_{1}$, the center of this circle is

$$
x=t_{1}+t_{2}+t_{3}+t_{4}=S_{1} .
$$

Since $S_{4}=t_{1} t_{2} t_{3} t_{4}=1$, the center is given by

or

$$
x=t_{1}+t_{2}+t_{3}+\frac{1}{t_{1} t_{2} t_{3}}
$$

$$
x=S_{1}+\frac{1}{S_{3}},
$$

where the $S$ 's refer now to three $t$ 's. But since the circle touches all four common lines there are three other expressions similar to (4) giving each the center of this circle.

If we write*

$$
x=S_{1}-t+t / S_{4}
$$

where now $S_{1}$ and $S_{4}$ refer to three of the parameters above and any fourth parameter, and not merely to those of the four common lines, we have the equation of a circle whose center is $S_{1}$ and whose radius is $\left|1-1 / S_{4}\right|$. It is the circle on the four equidistant points of the four triangles of three out of four directed lines.

It may be easily shown directly that (4) is the center of a circle on three

"This process may be called "symmetrizing." 
lines of the $A^{4}$. The equidistant axis of two of its lines with respective parameters $t_{1}$ and $t_{2}$ is

or

$$
t_{1} x+\frac{\bar{x}}{t_{1}}-\left(t_{1}^{2}+\frac{1}{t_{1}^{2}}+\mu\right)-t_{2} x+\frac{\bar{x}}{t_{2}}-\left(t_{2}^{2}+\frac{1}{t_{2}^{2}}+\mu\right)=0,
$$

Similarly

$$
x+\frac{1}{t_{1} t_{2}} \bar{x}=\left(t_{1}^{2}+\frac{1}{t_{1}^{2}}\right)-\left(t_{2}^{2}+\frac{1}{t_{2}^{2}}\right)
$$

$$
x+\frac{1}{t_{1} t_{3}} \bar{x}=\left(t_{1}^{2}+\frac{1}{t_{1}^{2}}\right)-\left(t_{3}^{2}+\frac{1}{t_{3}^{2}}\right)
$$

is the equation of a second one of these three axes. These two axes meet at the point

$$
x=\frac{t_{1}\left(t_{2}-t_{3}\right)\left(t_{1}^{2}+1 / t_{1}^{2}\right)-t_{2}\left(t_{1}-t_{3}\right)\left(t_{2}^{2}+1 / t_{2}^{2}\right)+t_{3}\left(t_{1}-t_{2}\right)\left(t_{3}+1 / t_{3}^{2}\right)}{\left(t_{1}-t_{2}\right)\left(t_{1}-t_{3}\right)\left(t_{2}-t_{3}\right)},
$$

which reduces at once to

and from this we obtain

$$
x=t_{1}+t_{2}+t_{3}+\frac{1}{t_{1} t_{2} t_{3}}
$$

$$
x=S_{1}-t+\frac{t}{S_{4}}
$$

( $S$ 's for 4 's ) ,

the same circle as before, thus verifying the result obtained by combining the line equations of the two curves above. This circle may in future be referred to as the "first" or center* circle of four directed lines.

Let us consider three directed lines of the $A^{4}$ given by parameters $t_{1}, t_{2}, t_{3}$. A fourth line with parameter $t_{4}$ is

$$
t_{4}^{4}-t_{4}^{3} x+\mu t_{4}^{2}-t_{4} \bar{x}+1=0 .
$$

A perpendicular to this line is of form

$$
t_{4}^{2} x-\bar{x}=k,
$$

and if this perpendicular is to be on the point equidistant from the three lines of the $A^{4}$ given, say, by the parameters $t_{1}, t_{2}, t_{3}$, that is, on the point

we have

$$
S_{1}+\frac{1}{S_{3}}
$$

$$
t_{4}^{2} x-\bar{x}=t_{4}^{2}\left(S_{1}+\frac{1}{S_{3}}\right)-\left(\frac{S_{2}}{S_{3}}+S_{3}\right),
$$

* Loud, these Transactions, vol. 1 (1900), p. 325, Theorem I. 
since the conjugate of $S_{1} \equiv t_{1}+t_{2}+t_{3}$ is

$$
\frac{1}{t_{1}}+\frac{1}{t_{2}}+\frac{1}{t_{3}}=\frac{t_{2} t_{3}+t_{3} t_{1}+t_{1} t_{2}}{t_{1} t_{2} t_{3}}=\frac{S_{2}}{S_{3}}
$$

and that of $S_{3}$ is $1 / S_{3}$.

Consider the following equation:

$$
t^{2} x-\bar{x}=t^{2}\left(S_{1}-t+\frac{t}{S_{4}}\right)-\left[\frac{\left(S_{2}-S_{1} t+t^{2}\right) t}{S_{4}}\right]\left[S_{3}-S_{2} t+S_{1} t^{2}-t^{3}\right]
$$

When the parameter $t$ takes in succession the values $t_{1}, t_{2}, t_{3}, t_{4}$, this equation reduces to the equation of each of the four perpendiculars like that given by (6), a line for each of the four sets of three $t^{\prime}$ s selected from $t_{1}, t_{2}, t_{3}, t_{4}$. In other words we have in (7) the equation of the four perpendiculars from the four equidistant points of the triangle of 3 of 4 lines upon the line left out in each case. On simplifying (7) we have

$$
\left(x-\frac{S_{1}}{S_{4}}\right) t^{2}-\left(\bar{x}-S_{3}\right)=t\left(S_{2}-\frac{S_{2}}{S_{4}}\right),
$$

the line equation in conjugate coördinates of a circle. Hence, we have the first of a chain of theorems:

If from each of the four equidistant points of 3 of 4 directed lines, a perpendicular be let fall upon the line left out in each case, the four-perpendiculars are on a circle.

This circle may be called the second circle of a directed 4-line.

The center of this circle is $S_{1} / S_{4}$; its radius, $\frac{1}{2}\left|S_{2}\left(1-1 / S_{4}\right)\right|$. But the absolute value of $S_{1}$ is equal to that of $S_{1} / S_{4}$, since each is $\sqrt{ } S_{1} S_{3} / S_{4}$. Also, $S_{1}$ was the center found for the circle on the four equidistant points of the triangles of 3 of 4 lines, while the origin is the center of the $A^{4}$ as given by the equation

$$
t^{4}-t^{3} x+\mu t^{2}-t \bar{x}+1=0 .
$$

We have then the theorem, the first of a second chain:

The center of the $A^{4}$, of which four directed lines are given, is equidistant from the center of the circle on the four equidistant points of these lines and from the center of the circle touched by the perpendiculars, one each from the equidistant point of three of the lines to the remaining line.

As we have explained what circle the center circle is, we may restate the above theorem:

The center of the $A^{4}$, of which four directed lines are given, is equidistant from the centers of the "center" and "second" circles for these lines.

The line equation of the second circle of a 4-line was

$$
\left(x-\frac{S_{1}}{S_{4}}\right) t^{2}-\left(\bar{x}-S_{3}\right)=t\left(S_{2}-\frac{S_{2}}{S_{4}}\right) \text {. }
$$


Differentiating this equation as to $t$, and then calling the parameter $T$ instead of $t$, for convenience, we have the map, or point, equation of this circle:

$$
2 T\left(x-\frac{S_{1}}{S_{4}}\right)=\left(S_{2}-\frac{S_{2}}{S_{4}}\right) .
$$

On symmetrizing this equation, there results

$$
2 T\left(x-\frac{\left(S_{1}-t\right) t}{S_{5}}\right)=\left(S_{2}-S_{1} t+t^{2}\right)\left(1-\frac{t}{S_{5}}\right),
$$

where the $S$ 's are for $5 t$ 's.

Now five $t$ 's are connected by the relation

or

$$
t^{5}-S_{1} t^{4}+S_{2} t^{3}-S_{3} t^{2}+S_{4} t-S_{5}=0
$$

$$
S_{2} t-S_{1} t^{2}+t^{3}=S_{3}-\frac{S_{4}}{t}+\frac{S_{5}}{t^{2}}
$$

Hence (10) may be written as

$$
x=\frac{\left(S_{1}-t\right) t}{S_{5}}+\frac{1}{2 T}\left[\left(S_{2}-S_{1} t+t^{2}\right)-\frac{1}{S_{5}}\left(S_{3}-\frac{S_{4}}{t}+\frac{S_{5}}{t^{2}}\right)\right] .
$$

If in this equation $t$ takes the value $t_{5}$, we obtain

$$
x=\frac{S_{1}}{S_{4}}+\frac{1}{2 T}\left(S_{2}-\frac{S_{2}}{S_{4}}\right),
$$

where the $S$ 's refer to $t_{1}, t_{2}, t_{3}, t_{4}$; and if $t$ should take the value $t_{4}$, we likewise obtain

$$
x=\frac{S_{1}}{S_{4}}+\frac{1}{2 T}\left(S_{2}-\frac{S_{2}}{S_{4}}\right),
$$

but here the $S$ 's now refer to $t_{1}, t_{2}, t_{3}, t_{5}$.

Similarly for $t$ taking any of the values $t_{1}, t_{2}$, or $t_{3}$. Hence when $t$ takes in succession the values $t_{5}, t_{4}, t_{3}, t_{2}, t_{1}$, (11) successively becomes the second circle for the sets of four lines with parameters $t_{1}, t_{2}, t_{3}, t_{4} ; t_{1}, t_{2}, t_{3}, t_{5}$; $t_{1}, t_{2}, t_{4}, t_{5} ; t_{1}, t_{3}, t_{4}, t_{5} ; t_{2}, t_{3}, t_{4}, t_{5}$ respectively. In other words (11) gives for the proper values of $t$ the five second circles of a directed 5-line, one for each of the 4-lines contained in the 5-line. Moreover, since five lines uniquely determine an $A^{4}$, any five lines with clinants of form $-1 / t^{2}$ are lines of an $A^{4}$ as written above.

Again, (11) not only contains the five second circles of a directed 5-line, but depends upon two independent parameters $t$ and $T$, both of absolute value unity. If in it we hold $t$ and let $T$ vary, it is the equation of a circle (as we have just seen for particular values of $t$ ); if we hold $T$ and let $t$ vary, it is the equation of a quartic curve. Thus, for all values of $t, \mathrm{~T}$ being the 
variable, there is a system of circles; and for all values of $T, t$ being the variable, there is a system of quartic curves. These two systems of curves, given by one equation, have the same envelope. In general, in the method of analysis employed in this paper, the condition for an envelope is

$$
\frac{t D_{t} f}{T D_{T} f}=\text { its conjugate }
$$

for equations of the form $x=f(t, T)$. If we now apply this condition to the present equation, we may be able to obtain the envelope of the system of circles it represents for a variable $T$ and fixed $t$ 's.

Differentiating (11) as to $t$ and multiplying the result by $t$, we have

$$
t D_{t} x=\frac{\left(S_{1}-2 t\right) t}{S_{5}}+\frac{1}{2 T}\left[\left(-S_{1}+2 t\right) t-\frac{1}{S_{5}}\left(\frac{S_{4}}{t}-\frac{2 S_{5}}{t^{2}}\right)\right] .
$$

Again, differentiating (11) as to $T$ and multiplying the result by $T$, we have

$$
T D_{T} x=-\frac{1}{2 T}\left[\left(S_{2}-S_{1} t+t^{2}\right)-\frac{1}{S_{5}}\left(S_{3}-\frac{S_{4}}{t}+\frac{S_{5}}{t^{2}}\right)\right] .
$$

The conjugate of $t D_{t} x$ is

$$
\left(\frac{S_{4}}{t}-\frac{2 S_{5}}{t^{2}}\right)+\frac{1}{2} T\left[\left(-\frac{S_{4}}{S_{5} t}+\frac{2}{t^{2}}\right)-\left(S_{1} t-2 t^{2}\right)\right],
$$

while that of $T D_{T} x$ is

$$
-\frac{1}{2} T\left[\left(\frac{S_{3}}{S_{5}}-\frac{S_{4}}{S_{5}}+\frac{1}{t^{2}}\right)-\left(S_{2}-S_{1} t+t^{2}\right)\right] .
$$

Substitution of these expressions in

gives, after reduction,

$$
\frac{t D_{r} x}{T D} \frac{x}{T} x=\text { its conjugate }
$$

$$
\begin{aligned}
\frac{\left(S_{1}-2 t\right) t T}{S_{5}}+\frac{1}{2}[ & \left.\left(-S_{1} t+2 t^{2}\right)-\left(\frac{S_{4}}{S_{5}^{3} t}-\frac{2}{t^{2}}\right)\right] \\
& +\frac{1}{t T}\left(S_{4}-\frac{2 S_{5}}{t}\right)+\frac{1}{2}\left[\left(-\frac{S_{4}}{S_{5} t}+\frac{2}{t^{2}}\right)-\left(S_{1} t-2 t^{2}\right)\right]=0,
\end{aligned}
$$

which may be written in the following form

$$
\left(\frac{S_{1}-2 t}{S_{5}}\right) t T+\frac{1}{t T}\left(S_{4}-\frac{2 S_{5}}{t}\right)-t\left(S_{1}-2 t\right)-\frac{1}{t}\left(\frac{S_{4}}{S_{5}}-\frac{2}{t}\right)=0
$$

This equation is a quadratic in $T$, of which one root is evidently $T=S_{5}$. 
The other root is

$$
\frac{S_{4} t-2 S_{5}}{t^{3}\left(S_{1}-2 t\right)}
$$

whose conjugate is its reciprocal. Hence both roots are turns.

If now these values be put in (11) the resulting equation in $t$ will give the envelope of the system of circles corresponding to the roots substituted. Consider that part of the envelope obtained by putting $T=S_{5}$ in (11). We then have

$$
x=\frac{S_{2}-S_{1} t-t^{2}}{2 S_{5}}-\frac{1}{2 S_{5}^{2}}\left(S_{3}-\frac{S_{4}}{t}+\frac{S_{5}}{t^{2}}\right),
$$

the envelope expressed parametrically. We can obtain the equation of the envelope free of the parameter $t$ by eliminating it between (13) and its conjugate. The resulting equation will be expressed of course entirely by means of conjugate coördinates. Now the conjugate of (13) is

$$
\bar{x}=\frac{1}{2}\left(S_{3}+\frac{S_{4}}{t}-\frac{S_{5}}{t^{2}}\right)-\frac{1}{2} S_{5}^{2}\left(\frac{S_{2}}{S_{5}}-\frac{S_{1} t}{S_{5}}+\frac{t^{2}}{S_{5}}\right) .
$$

Combining (13) and (14) so as to eliminate $t$, we have

$$
x-\frac{\bar{x}}{S_{5}^{2}}=\frac{S_{2}}{S_{5}}-\frac{S_{3}}{S_{5}^{2}},
$$

the equation of a line. Thus we have another theorem of the first chain:

The five second circles of four out of five directed lines are on a line.

The second part of the envelope of this system of circles is of course obtained by putting the second root of the quadratic in $T$ above, viz.,

in (11). Whence we have

$$
\frac{S_{4} t-2 S_{5}}{t^{4}\left(S_{1}-2 t\right)}
$$

$$
x=\frac{\left(S_{1}-t\right) t}{S_{5}}+\frac{t^{4}\left(S_{1}-2 t\right)}{2\left(S_{4} t-2 S_{5}\right)}\left[\left(S_{2}-S_{1} t+t^{2}\right)-\frac{1}{S_{5}}\left(S_{3}-\frac{S_{4}}{t}+\frac{S_{5}}{t^{2}}\right)\right]
$$

the map equation of a curve of the 15th degree. This curve is not considered in this article.

82. Second circle for a directed 2n-line. Directrix for a directed $2 n+1$-line.

In the preceding section the parallel to the hypocycloid of class 4 , the curve which we have termed the $A^{4}$, was used for the metrical treatment of the directed 5-line, the directed 4-line being considered as a special case of the 5-line. For a like treatment of the 7-line, the curve $A^{6}$, also mentioned in the preceding section, may be used, and the 6 -line considered as a special case of the 7-line. It can be shown in fact that 
(a) If from each center of the $A^{4}$, inscribed in 5 of 6 lines of direction, a perpendicular be let fall upon the line omitted in each case, the 6 perpendiculars are on a circle, the second circle of a 6-line.

(b) The 7 second circles of 6 out of 7 lines of direction are on a line, the directrix of a 7-line.

And so on in general.

Hence, the most general curve of the same type as the $A$ curves is at once suggested for the metrical handling of $2 n+1$ lines. It may be written

$$
\begin{aligned}
(-)^{n}\left(x t^{n+1}+\bar{x} t^{n-1}\right)=t^{2 n}+1-\left(S_{1} t^{2 n-1}+S_{2 n-1} t\right)+\cdots+(-)^{n} \mu t^{n} \\
+\cdots+(-)^{n}\left(S_{n-2} t^{n+2}+S_{n+2} t^{n-2}\right) .
\end{aligned}
$$

This is the line equation of the curve. The coefficients $S_{a}$ and $S_{2 n-a}$ are conjugates and $\mu$ is real. For a given $x$ there are $2 n$ values of $t$, that is, the curve is of class $2 n$. On substituting the values of $x$ in the map * equation (given further on) and its conjugate in the equation of a line

$$
a x+\bar{a} \bar{x}+1=0 \text {, }
$$

it is seen that the curve is of order $2 n+2$. By the aid of the curves, $A^{2 n}$ and $A^{2 n-2}$, the general theorems of the two chains of theorems already indicated may be proved.

Consider, then, the $A^{2 n}$ in the form

$$
t^{2 n}-a_{1} t^{2 n-1}+\cdots+(-)^{n+1} x t^{n+1}+(-)^{n} \mu t^{n}+(-)^{n-1} t^{n-1}
$$

and also the general $A^{2 n-2}$ as given by

$$
+(-)^{n-2} \cdots-a_{2 n-1} t+1=0
$$

$$
\begin{aligned}
& \alpha t^{2 n-2}-\beta t^{2 n-3}+\gamma t^{2 n-4}-\cdots+(-)^{n}(x-\delta) t^{n}+(-)^{n-1} \mu_{1} t^{n-1} \\
& \quad+(-)^{n}(x-\kappa) t^{n-2}+(-)^{n-1} \lambda t^{n-3}+(-)^{n} \cdots-\xi t+\zeta=0 .
\end{aligned}
$$

Eliminating $x$ and $\bar{x}$ from (16) by combining with it (17), we have

$$
\begin{aligned}
t^{2 n}- & a_{1} t^{2 n-1}+\cdots-a_{2 n-1} t+1+\left[(-)^{n} \mu+(-)^{n-1} \mu_{1}\right] t^{2}+\cdots \\
& +t\left[\alpha t^{2 n-2}-\beta t^{2 n-3}+\gamma t^{2 n-4}-\cdots(-)^{n+1} \delta t^{n}+(-)^{n+1} \kappa t^{n-2}+\cdots-\lambda t+\nu\right]=0,
\end{aligned}
$$

an equation whose $2 n$ roots are the parameters of $2 n$ common lines of direction of the $A^{2 n}$ and the $A^{2 n-2}$. The center of the $A^{2 n-2}$ is

$$
\delta=\Sigma t_{1} t_{2} t_{3} \cdots t_{n-1} \equiv S_{n-1} \text {. }
$$

It is the center for the reason that, on obtaining the map equation of the $A^{2 n-2}$, the term containing $\delta$ is the only one not containing $t$ when the equation is solved for $x$. This is in accordance with the definition of the $A$ curves pre-

* Equation $(E)$ near the end of this section. 
viously given. Also, $S_{n-1}$ here belongs to $2 n t$ 's; and these are connected by the characteristic relation

$$
S_{2 n}=1 \text {. }
$$

Now, inasmuch as the center of the $A^{2 n-2}$ is $S_{n-1}$, and since here $S_{2 n}=1$, the center of the $A^{2 n-2}$ on any $2 n-1$ lines of the $2 n$ common lines of the two curves is

in which the $S$ 's now are for $2 n-1 t$ 's.

$$
x=S_{n-1}+\frac{S_{n-2}}{S_{2 n-1}},
$$

A perpendicular to a line of the $A^{2 n}$ with parameter $t_{2 n}$ and on the center of the $A^{2 n-2}$ just given is

$$
t_{2 n}^{2} x-\bar{x}=t_{2 n}^{2}\left(S_{n-1}+\frac{S_{n-2}}{S_{2 n-1}}\right)-\left(\frac{S_{n}}{S_{2 n-1}}+S_{n+1}\right) .
$$

If this equation be now written for $2 n t^{\prime}$ s, it becomes

$$
\begin{aligned}
x t^{2}-\bar{x}=t^{2}\left[S_{n-1}-S_{n-2} t\right. & +\cdots+(-)^{n-1} t^{n-1} \\
& \left.+\frac{\left(S_{n-2}-S_{n-3} t+\cdots+(-)^{n-2} t^{n-2}\right) t}{S_{2 n}}\right] \\
& -\left[\frac{t\left(S_{n}-S_{n-1} t+\cdots+(-)^{n} t^{n}\right)}{S_{2 n}}\right] \\
& -\left[S_{n+1}-S_{n} t+S_{n-1} t^{2}-\cdots+(-)^{n+1} t^{n+1}\right] .
\end{aligned}
$$

This reduces to

$$
t^{2}\left(x-\frac{S_{n-1}}{S_{2 n}}\right)-\left(\bar{x}-S_{n+1}\right)=t\left(S_{n}-\frac{S_{n}}{S_{2 n}}\right),
$$

the equation of a circle in line form. This circle is touched by every one of the $2 n$ perpendiculars contained in (18), there being one perpendicular for each $t_{i}, i$ running from 1 to $2 n$. Whence we have the general theorem:

If from each center of the $A^{2 n-2}$ on every $2 n-1$ out of $2 n$ lines of direction of an $A^{2 n}$, a perpendicular be let fall upon the line left out in each case, the $2 n$ perpendiculars are on a circle.

This may be termed the second circle of a $2 n$-line.

The point equation of this circle is

$$
x=\frac{S_{n-1}}{S_{2 n}}+\frac{1}{2 T}\left(S_{n}-\frac{S_{n}}{S_{2 n}}\right) .
$$

For $2 n+1 t$ 's this equation becomes

$$
\begin{aligned}
x=\frac{S_{n-1} t-S_{n-2} t^{2}+\cdots+(-)^{n-1} t^{n}}{S_{2 n+1}}+\frac{1}{2 T}\left[\left(S_{n}-S_{n-1} t+\cdots\right.\right. \\
\left.\left.+(-)^{n} t^{n}-\frac{1}{S_{2 n+1}}\right)\left(S_{n+1}-\frac{S_{n+2}}{t}+\cdots+(-)^{n} \frac{S_{2 n+1}}{t^{n}}\right)\right] .
\end{aligned}
$$


If in (21), $t$ takes the values $t_{i}(i=1,2, \cdots n+1)$, the equation reduces to the corresponding second circle for $2 n$ out of $2 n+1$ lines. If $t$ be any constant of the unit circle, and if $T$ varies, there is also a circle. Hence for all constants of the unit circle there is a single infinity of circles to which system the above second circles belong. What is the envelope of this system? If we obtain from (21) $t D_{t} x$ and $T D_{T} x$, take their conjugates and substitute in the equation of condition for contact, viz.,

$$
\frac{t D_{t} x}{T D_{T} x}=\text { its conjugate, }
$$

we have, after reduction,

$$
\begin{aligned}
\frac{\left(S_{n-1}-2 S_{n-2} t+\cdots+(\right.}{S_{2 n+1}} & \\
& +\frac{1}{t T}\left(S_{n+2} n t^{n-1}\right) t T \\
& -\left(S_{n-1} t-2 S_{n-2} t^{2}+\cdots+(-)^{n-1} n t^{n}\right) \\
& -\frac{1}{S_{n+1}}\left(\frac{S_{n+2}}{t}-\frac{2 S_{2 n}}{t^{2}}+\cdots+(-)^{n-1} \frac{n S_{2 n+1}}{t^{n}}\right)=0,
\end{aligned}
$$

a quadratic in $T$ whose two roots are

and

$$
T_{1}=S_{2 n+1}
$$

$$
T_{2}=\frac{S_{n+2} t^{n-1}-2 S_{2 n} t^{n-2}+\cdots+(-)^{n-1} n S_{2 n+1}}{S_{n-1}-2 S_{n-2} t+\cdots+(-)^{n-1} n t^{n-1}}
$$

both of which are turns. If we put $T_{1}=S_{2 n}+1$ in (21) we find

$$
x=\frac{S_{n}+S_{n-1} t-\cdots+(-)^{n-1} t^{n}}{2 S_{2 n+1}}-\frac{1}{2 S_{2 n+1}^{2}}\left(S_{n+1}-\frac{S_{n+2}}{t}+\cdots(-)^{n} \frac{S_{2 n+1}}{t^{n}}\right) .
$$

Combining this value of $x$ with its conjugate, we have

$$
x-\frac{1}{S_{2 n+1}^{2}} \bar{x}=\frac{S_{n}}{S_{2 n+1}}-\frac{S_{n+1}}{S_{2 n+1}^{2}},
$$

the equation of a line. Hence, the general theorem:

The $2 n+1$ second circles of $2 n$ out of $2 n+1$ directed lines are on a line.

It has been shown that the center of the $A^{4}$ of which four lines are given is equally distant from the center of the center and second circles for these lines. There are similar theorems for a greater number of lines. The center of the $A^{4}$ on 5 of a set of 6 common lines of the $A^{4}$ and $A^{6}$ is

$$
x=S_{2}+S_{1} / S_{6}
$$


where the $S$ 's are for $5 t$ 's. For 6 lines

$$
x=S_{2}-S_{1} t+t^{2}+\left(S_{1}-t\right) t / S_{6},
$$

the equation of a limaçon with center $S_{2}$. Hence the theorem:

The 6 centers of ihe $A^{4}$ 's of 5 of 6 lines of the $A^{6}$ are on a limaçon whose center is $S_{2}$.

As 5 points, in general, determine uniquely a limaçon, these 6 points are not any 6 points of the plane, but are connected by the relation just deduced. But the absolute value of the stroke giving the center of the second circle for 6 lines was $\left|S_{2} / S_{6}\right|$. Now,

$$
\left|S_{2} / S_{6}\right|=\left|S_{2}\right|
$$

whence follows the theorem:

The center of the $A^{6}$, of which 6 lines are given, is equally distant from the center of the limaçon on which are the centers of the $6 A^{4}$ 's on 5 of 6 common lines of the $A^{4}$ and $A^{6}$, and from the center of the second circle for the 6 lines.

These theorems put generally are, therefore,

First, The $2 n$ centers of the $A^{2 n-2}$ 's on $2 n-1$ of $2 n$ lines of direction are on the trochoidal curve

$$
\begin{aligned}
x=S_{n-1}-S_{n-2} t+\cdots+(-)^{n-1} t^{n-1}+ & \left(S_{n-2}-S_{n-3} t\right. \\
& \left.+\cdots+(-)^{n} t^{n-2}\right) t / S_{2 n} .
\end{aligned}
$$

Also, since the center of the second circle for $2 n$ lines is $S_{n-1} / S_{2 n}$, while that of the curve just given is $S_{n-1}$, we have

Secondly, The center of the $A^{2 n}$ of which $2 n$ lines are given is equally distant from the center of the second circle for $2 n$ lines of the $A^{2 n}$ and from the center of the curve of centers of the $A^{2 n-2}$ 's of $2 n-1$ of $2 n$ lines.

If in the equation of the $A^{2 n}$, viz., $t^{2 n}-S_{1} t^{2 n-1}+\cdots+(-)^{n+1} x t^{n+1}+(-)^{n} \mu t^{n}+(-)^{n-1} \bar{x} t^{n-1}+\cdots$

we make $n=3$, we have the equation of the $A^{6}$

$$
\cdots-S_{2 n-1} t+1=0 \text {, }
$$

$$
t^{6}-S_{1} t^{5}+x t^{4}-\mu t^{3}+\bar{x} t^{2}-S_{5} t+1=0 .
$$

The map equation is

$$
x=-2 t^{2}+\frac{3}{2} S_{1} t+\mu / 2 t-S_{5} / 2 t^{3}+1 / t^{4} .
$$

Differentiation as to $t$ gives

$$
0=-8 t+3 S_{1}-\mu / t^{2}+3 S_{5} / t^{4}-8 / t^{5},
$$

an equation whose 6 roots are the 6 parameters of the cusps of the $A^{6}$. That 
is, the cusps are given by the roots of

$$
8\left(t^{6}+1\right)+\mu t^{3}-3\left(S_{1} t^{5}+S_{5} t\right)=0 .
$$

Now, combining the line equation of the $A^{6}$ with this cusp condition, we have

$$
8\left(x t^{3}+\bar{x} t\right)-5\left(S_{1} t^{4}+S_{5}\right)-9 \mu t^{2}=0 .
$$

This is the equation of an $A^{4}$ concentric with the $A^{6}$. Hence,

The 6 cusp tangents of an $A^{6}$ touch a concentric $A^{4}$.

The map equation of the $A^{2 n}$ is

$$
\begin{array}{r}
(-)^{n}(2 x)=\left[(n+1) t^{n-1}-\frac{n-1}{t^{n+1}}\right]-\left[S_{1} n t^{n-2}-\frac{(n-2) S_{2 n-1}}{t^{n}}\right] \\
+\cdots+(-)^{n} \frac{\mu}{t} \cdots+(-)^{n}\left(3 S_{n-2} t-\frac{S_{n+2}}{t^{3}}\right) .
\end{array}
$$

The equation giving the cusp condition is

$$
\begin{array}{r}
0=(n+1)(n-1)\left(t^{2 n}+1\right)-\left[S_{1} n(n-2) t^{2 n-1}+S_{2 n-1} n(n-2) t\right] \\
+\cdots+(-)^{n} \mu t^{n}+\cdots+(-)^{n}\left[3 S_{n-2} t^{n+2}+3 S_{n+2} t^{n-2}\right] .
\end{array}
$$

This combined with the line equation gives

$$
\begin{aligned}
(-)^{n}(n+1)(n+2)\left(x t^{n}+\bar{x} t^{n-2}\right) & =(1-2 n)\left[S_{1} t^{2 n-2}+S_{2 n-1}\right] \\
& +\cdots+(-)^{n} n^{2} \mu t^{n-1}+\cdots+(-)^{n}\left(n^{2}-2\right)\left[S_{n-2} t^{n+1}+S_{n+2} t^{n-3}\right] .
\end{aligned}
$$

But this is the equation of an $A^{2 n-2}$ concentric with the given $A^{2 n}$. Therefore,

There are $2 n$ cusp tangents of an $A^{2 n}$ and these tangents touch a concentric $A^{2 n-2}$.

\section{§3. Reversal of direction.}

The 4-line.

The standard equation of a line to which a direction may be assigned, viz.,

$$
t x+\frac{\bar{x}}{t}-2 r=0,
$$

is adapted to the analytical discussion of reversal of direction.

The equidistant axis of two lines with parameters $t_{1}$ and $t_{2}$ respectively was

or

$$
\left(t_{1} x+\frac{\bar{x}}{t_{1}}-2 r_{1}\right)-\left(t_{2} x+\frac{\bar{x}}{t_{2}}-2 r_{2}\right)=0,
$$

$$
x-\frac{1}{t_{1} t_{2}} \bar{x}=\frac{2\left(r_{1}-r_{2}\right)}{t_{1}-t_{2}} .
$$


The meet of these two lines is

$$
x=\frac{2 r_{1} t_{1}-2 r_{2} t_{2}}{t_{1}^{2}-t_{2}^{2}} .
$$

A perpendicular to the equidistant axis and on the point of meeting just given is

$$
x+\frac{1}{t_{1} t_{2}} \bar{x}=\frac{2\left(r_{1}+r_{2}\right)}{t_{1}+t_{2}},
$$

a result immediately obtained from (25a) if in it we change the sign of both $r_{2}$ and $t_{2}$. But this is precisely what is also obtained from the equidistant axis, if in it the sign of the second parenthesis is changed from - to + . Now, from the discussion of a pair of directed lines, we recall that the effect of reversing the direction of one of a pair of lines is to pass from one bisector of the angles at the intersection of the lines to the other.* Hence the effect of a reversal of the direction of one of the lines may be expressed analytically in the formulas of directed lines simply by changing the sign of the corresponding $t$ and $r$ of that line. And this rule applies to the reversal of the direction of any number of lines.

A directed 4-line is best studied when the lines are taken as lines of a parabola, one of whose defining properties is, that the reflection of the focus in any tangent is on a line. Let this line be

$$
y=\frac{1}{1-t}
$$

which, on elimination of $t$ between the given equation and its conjugate, becomes

Let a particular point on it be

$$
y+\bar{y}=1
$$

$$
y_{1}=\frac{1}{1-t_{1}}
$$

This is the reflexion of the focus in a tangent. Then the current coördinate $x$ of a point on a tangent and $y_{1}$ are connected by the relation

$$
x-y_{1}=t x .
$$

Hence all tangents of the parabola are given by

$$
x=\frac{1}{(1-t)\left(1-t_{1}\right)} ;
$$

also for $t_{1}=t$, we have the map equation of the parabola itself

$$
x=\frac{1}{(1-t)^{2}},
$$

\footnotetext{
* See Introduction.
}

Trans. Am. Math. Soc. 14 
or replacing $t$ by $t^{2}$

$$
x=\frac{1}{\left(1-t^{2}\right)^{2}} \text {. }
$$

The corresponding line equation is

$$
t x+\frac{\bar{x}}{t}-\frac{t}{1-t^{2}}=0
$$

which is of form suited to giving the line a specified direction. Corresponding to (26), however, a line of the parabola is

$$
t x-\bar{x}=\frac{t}{1-t} .
$$

We do not give a direction to this line; and it is evident that with every directed tangent with clinant $1 / t^{2}$, there are two associated undirected tangents with clinants respectively equal to $1 / t$ and $-1 / t$. These two lines intersect on the directrix of the parabola.

The osculant* form of (26), viz.,

$$
x=\frac{1}{\left(1-t_{1}\right)\left(1-t_{2}\right)}
$$

is the meet of two lines of the curve with parameters $t_{1}$ and $t_{2}$. The circumcircle of three such undirected lines is, therefore,

$$
x=\frac{1-t}{\left(1-t_{1}\right)\left(1-t_{2}\right)\left(1-t_{3}\right)} \equiv \frac{1-t}{A},
$$

while that for the three lines with parameters $-t_{1},-t_{2},-t_{3}$ is

$$
x=\frac{1+t}{\left(1+t_{1}\right)\left(1+t_{2}\right)\left(1+t_{3}\right)} \equiv \frac{1+t}{B} .
$$

The two respective center circles for the corresponding sets of four lines are

and

$$
x=\frac{1-t}{\left(1-t_{1}\right)\left(1-t_{2}\right)\left(1-t_{3}\right)\left(1-t_{4}\right)} \equiv \frac{1-t}{A}
$$

$$
x=\frac{1+t}{\left(1+t_{1}\right)\left(1+t_{2}\right)\left(1+t_{3}\right)\left(1+t_{4}\right)} \equiv \frac{1+t}{B}
$$

with centers respectively at the points

$$
x=\frac{1}{A}
$$

( $A$ for four factors),

and

$$
x=\frac{1}{B} \quad \text { (B for four factors). }
$$

* E. Strdr, Leipziger Berichte (1886), p. 3; F. MorLer, these Transactions, vol. 1 (1900), p. 97. 
These circles are evidently on the origin for $t=1$ in the one case and $t=-1$ in the other. Moreover, their centers and the origin are on a line, for

$$
\left|\begin{array}{ccc}
\frac{1}{A} & \frac{S_{4}}{A} & 1 \\
\frac{1}{B} & \frac{S_{4}}{B} & 1 \\
0 & 0 & 1
\end{array}\right|=0 .
$$

Hence, for four directed lines of a parabola, the two center circles of associated undirected lines whose paramaters are $\pm t_{i}(i=1,2,3,4)$ are on the origin and have contact at the origin.

Similar work shows that the same thing is true for the seven additional pairs of center circles that can be selected from the two sets of four lines each. A pair of circles would be given, for instance, by $t_{1}, t_{2}, t_{3},-t_{4}$ and $-t_{1},-t_{2}$, $-t_{3}, t_{4}$. Of the eight pairs of these circles, four have as their common tangent at the origin

and the remaining four have

$$
S_{4} x+x=0,
$$

$$
S_{4} x-\bar{x}=0 .
$$

Thus, of the sixteen circles eight belong to one parabolic* system and eight to another; and every circle of one system intersects every circle of the other system orthogonally.

Consider next the circles on the equidistant points of four directed lines of a parabola. Arbitrarily assign directions to these lines. There is one corresponding circle on the four equidistant points. When, however, the lines with parameters, say, $t_{1}, t_{2}, t_{3}, t_{4}$ are reversed in all possible ways there are seven additional circles, four for a reversal of one line at a time and three for two at a time. Note that to reverse lines 3 and 4 is the same as to reverse 1 and 2 and that to reverse 2,3 , and 4 is the equivalent of the reversal of 1 . Thus, there are eight circles in all. As shown by Loud, $\uparrow$ these are divided into two orthogonal sets of four.

Now, in order to have four circles on two points and four circles about those points, it requires four constants to fix the two points, four more for the circles on the two points, and still four more for the circles about the points, making in all twelve constants; but the two orthogonal sets of circles are determined uniquely by four lines for which there are eight constants. It is evident, therefore, that these sets of circles are subject to relations. These we seek to find.

* HaRkness and MoRLey, Introduction to the Theory of Analytic Functions, p. 31.

† These Transactions, vol. 1 (1900), p. 335. 
The equidistant point of three directed lines of a parabola with clinants $1 / t_{1}^{2}, 1 / t_{2}^{2}, 1 / t_{3}^{2}$, is

$$
x=\frac{1+t_{1} t_{2}+t_{1} t_{3}+t_{2} t_{3}}{\left(1-t_{1}^{2}\right)\left(1-t_{2}^{2}\right)\left(1-t_{3}^{2}\right)} .
$$

Consequently, for four lines the equation,

$$
\left.x=\frac{1+S_{2}+S_{4}-\left(S_{1}+S_{3}\right) t}{P} \quad \text { ( } S^{\prime} \mathrm{s} \text { for four } t^{\prime} \mathrm{s}\right),
$$

where $P$ is the product $\left(1-t_{1}^{2}\right)\left(1-t_{2}^{2}\right)\left(1-t_{3}^{2}\right)\left(1-t_{4}^{2}\right)$, is the center circle of the four lines with their original directions. But suppose the fourth line is reversed. What then is the equation of this circle?

The line of the parabola to which we assign direction, viz.;

can be used as is

$$
t x-\frac{\bar{x}}{t}=\frac{t}{1-t^{2}}
$$

$$
t x+\frac{\bar{x}}{t}=2 r,
$$

the $t /\left(1-t^{2}\right)$ in the former taking the place of $2 r$ in the latter. When we reverse a line given by the latter, we change, as we saw, the corresponding $t$ and $r$. For a line of the parabola, however, a change of only the corresponding $t$ is necessary, since a change of sign of $t$ changes in this line also the sign of what corresponds to $r$ in the standard equation of the line. And this conclusion is directly verified in all the formulas used. Hence the equidistant point for lines, 1, 2, and 4 with 4 reversed is

$$
x=\frac{1+t_{1} t_{2}-t_{1} t_{4}-t_{2} t_{4}}{\left(1-t_{1}^{2}\right)\left(1-t_{2}^{2}\right)\left(1-t_{3}^{2}\right)},
$$

and the center circle for $1,2,3$, and 4 reversed is

or

$$
x=\frac{\left(1+S_{2}^{4}-S_{1}^{4} t+t^{2}\right)\left(1-t^{2}\right)}{\left(1-t_{1}^{2}\right)\left(1-t_{2}^{2}\right)\left(1-t_{3}^{2}\right)\left(1-t_{4}^{2}\right)},
$$

$$
x=\frac{1+S_{2}^{4}+S_{4}^{4}-\left(S_{1}^{4}+S_{3}^{4}\right) t}{\left(1-t_{1}^{2}\right)\left(1-t_{2}^{2}\right)\left(1-t_{3}^{2}\right)\left(1-t_{4}^{2}\right)} .
$$

Similarly for 1 and 2 reversed simultaneously

$$
x=\frac{1+S_{2}^{12}+S_{4}^{12}-\left(S_{1}^{12}+S_{3}^{12}\right) t}{\left(1-t_{1}^{2}\right)\left(1-t_{2}^{2}\right)\left(1-t_{3}^{2}\right)\left(1-t_{4}^{2}\right)},
$$

in which $S_{i}^{4}$ and $S_{i}^{12}$ indicate the synmetric functions of the $t^{\prime}$ s when the signs have been changed. Denote these two circles by $C_{4}$ and $C_{12}$ respectively and 
the circle for the original directions by $C$. Of these eight circles, four belong to an elliptic* set-those obtained.by reversing two lines; four to a hyperbolic set-those obtained by reversing one line. Let us obtain the equation of the line of centers of the elliptic set. Two of these, $C$ and $C_{12}$ have centers

$$
x=\frac{1+S_{2}+S_{4}}{P}, \quad x=\frac{1+S_{2}^{12}+S_{4}^{12}}{P} .
$$

The line on these points is $S_{4} x-\bar{x}=0$. Similarly, the line of centers of the hyperbolic set is $S_{4} x+\bar{x}=0$. These two lines are perpendicular, as they should be.

Denote, for shortness, $1+S_{2}+S_{4}$ by $a$ and $S_{1}+S_{3}$ by $b$. Then the circle $C$ becomes

whence

$$
x=\frac{a-b t}{P}, \quad \bar{x}=\frac{(a-b / t) S_{4}}{P},
$$

Likewise $C_{1}$ is

$$
P^{2} x \bar{x}-P a\left(S_{4} x+\bar{x}\right)=S_{4}\left(b^{2}-a^{2}\right) .
$$

$$
P^{2} x \bar{x}-P a_{1}\left(-S_{4} x+\bar{x}\right)=-S_{4}\left(b_{1}^{2}-a_{1}^{2}\right) \cdot
$$

The line of centers $S_{4} x-\bar{x}=0$ intersects $C$ in the points $x=(a \pm b) / P$, and $S_{4} x+\bar{x}=0$ intersects $C_{1}$ in $x=\left(a_{1} \pm b_{1}\right) / P$.

The line on these two points is

$$
x-k \bar{x}=\frac{2 m n_{1}}{P\left(m+n_{1}\right)}, \quad k=\frac{m-n_{1}}{S_{4}\left(m+n_{1}\right)},
$$

in which $m=a+b$ and $n_{1}=a_{1}+b_{1}$. Again, the line on the points

has the clinant

$$
\frac{a_{14}+b_{14}}{P} \text { and } \quad \frac{a_{4}+b_{4}}{P}
$$

wheré $m_{14}=a_{14}+b_{14}$. Now

$$
\frac{m_{14}-n_{4}}{S_{4}\left(m_{14}+n_{4}\right)}
$$

$$
\begin{aligned}
m-n_{1} & =1+S_{2}+S_{4}+S_{1}+S_{3}-1-S_{2}^{1}-S_{4}^{1}-S_{1}^{1}-S_{3}^{1} \\
& =2 t_{1}\left(t_{2}+t_{3}+t_{4}\right)+2 t_{1}\left(t_{2} t_{3} t_{4}\right)+2 t_{1}+2 t_{1}\left(t_{2} t_{3}+t_{2} t_{4}+t_{3} t_{4}\right) \\
& =2 t_{1}\left(1+S_{1}+S_{2}+S_{3}\right) \quad\left(S^{\prime} \text { s for } t_{2}, t_{3}, t_{4}\right), \\
m_{14}+n_{4} & =2\left(1+S_{1}+S_{2}+S_{3}\right) .
\end{aligned}
$$

Consequently the clinant $k$ is equal to $\left(t_{2} t_{3} t_{4}\right)^{-1}$. In like manner, it is seen that the clinant of the line on the points $(a-b) / P$ and $\left(a_{1}-b_{1}\right) / P$ is $-\left(t_{2} t_{3} t_{4}\right)^{-1}$, while that of the line on $\left(a_{12}-b_{12}\right) / P$ and $\left(a_{2}-b_{2}\right) / P$ is

\footnotetext{
* Harkness aNd Morley, Introduction to the Theory of Analytic Functions, p. 31.
} 
$\left(t_{2} t_{3} t_{4}\right)^{-1}$, the former indicating a perpendicular, the latter a parallel, to the line whose equation has just been given.

Consider next one of the circles of the elliptic set, say, $C$. The line of centers

$$
S_{4} x-\bar{x}=0
$$

intersects this circle in two points. The companion line of centers $S_{4} x+\bar{x}=0$ intersects each of the circles $C_{1}, C_{2}, C_{3}, C_{4}$ in two points. Selecting $C_{1}$, say, the points of intersection are $\left(a_{1} \pm b_{1}\right) / P$. If now the intersections $(a \pm b) / P$ of $C$ are joined each to the eight points $\left(a_{i} \pm b_{i}\right) / P(i=1,2,3,4)$, there are sixteen lines, four for each circle $C_{i}$. One such set of four are lines on the points

$$
\begin{aligned}
& (a+b) / P \text { and }\left(a_{1} \pm b_{1}\right) / P, \\
& (a-b) / P \text { and }\left(a_{1} \pm b_{1}\right) / P,
\end{aligned}
$$

and have clinants respectively equal to

$$
\frac{1}{t_{2} t_{3} t_{4}}, \frac{S_{1}+S_{3}}{S_{4}\left(1+S_{2}\right)},-\frac{1}{t_{2} t_{3} t_{4}},-\frac{S_{1}+S_{3}}{S_{4}\left(1+S_{2}\right)}\left(S_{1}, S_{2}, S_{3} \text { for } t_{2}, t_{3}, t_{4}\right) \text {. }
$$

The four lines of course form a complete four-side; and the two pairs of perpendicular sides intersect at the orthogonal intersections of the two circles.

Again, if each circle of the elliptic set be joined in all ways to each circle of the hyperbolic set, in the manner indicated, there are 64 joining lines, and investigation shows that, of these lines, there are 4 sets of 4 parallels; 4 sets of 4 perpendiculars to these; 16 pairs of perpendicular lines.

These four sets of parallels may be considered as accounting for the four missing relations on the eight circles of the two orthogonal sets arising from the directed 4-line.

We had the line equation of the parabola in the form

$$
t x-\frac{\bar{x}}{t}=\frac{t}{1-t^{2}}
$$

with clinant $1 / t^{2}$. A perpendicular to this line and on the equidistant point of three lines with parameters $t_{1}, t_{2}, t_{3}$ is

$$
2\left(x+\frac{\bar{x}}{t^{2}}\right)=\frac{1}{A}+\frac{1}{B}-\frac{S_{4}}{t^{2}}\left(\frac{1}{A}-\frac{1}{B}\right) \quad(A, B \text { for } 3 \text { factors }) .
$$

This for four lines is

$$
2\left(x+\frac{x}{t^{2}}\right)=\frac{1-t}{A}-\frac{(1-t) S_{4} / t^{3}}{A}+\frac{1+t}{B}+\frac{(1+t) S_{4} / t^{3}}{B},
$$

the line equation of the second circle for four lines of direction of a parabola. 
The point equation is

$$
2 x=\frac{1-S_{1}}{A}+\frac{1+S_{1}}{B}+\frac{S_{2}}{2 t}\left(\frac{1}{A}-\frac{1}{B}\right),
$$

which gives for its center

$$
C=\frac{1}{2}\left(\frac{1-S_{1}}{A}+\frac{1+S_{1}}{B}\right) .
$$

If now we reverse the lines in all possible ways, we obtain seven additional second circles. For the reversal of line 3, say, we get the new circle

$$
2 x=\frac{1-S_{1}^{3}}{A_{3}}+\frac{1+S_{1}^{3}}{B_{3}}+\frac{S_{2}^{3}}{2 t}\left(\frac{1}{A_{3}}-\frac{1}{B_{3}}\right) ;
$$

for the reversal of lines 2 and 3 ,

$$
2 x=\frac{1-S_{1}^{23}}{A_{23}}+\frac{1+S_{1}^{23}}{B_{2 n}}+\frac{S_{2}^{23}}{2 t}\left(\frac{1}{A_{23}}-\frac{1}{B_{23}}\right) ;
$$

the notation of the last two indicating that in the $S$ 's, $A$ 's and $B$ 's the signs of the corresponding $t$ 's have been changed from + to - . This is all that is required to get the equations of the new circles, the equation of the original circle being given.

Consider next what is the simplest relation of their eight centers. The center of the original circle was:

$$
A B x=1+S_{2}+S_{4}-S_{1}\left(S_{1}+S_{3}\right) .
$$

For line 1 reversed; the center of the circle is

$$
A B x=1+S_{2}^{1}+S_{4}^{1}-S_{1}^{1}\left(S_{1}^{1}+S_{3}^{1}\right),
$$

and so on for the others. Note also that

$$
A B=A_{1} B_{1}=A_{12} B_{12} ;
$$

that is, a reversal of one or two lines does not affect $A B$. Also, $S_{4}$ is invariant for a change in sign of one $t$, and again for a change of sign of two simultaneously. If now from the equations of the various eight centers, all but one symmetric function of the t's be eliminated, we may be able to get a relation on the centers by interpreting properly the various functions of the remaining symmetric function considered as a parameter. Hitherto it has been convenient to use $t$ as a parameter, but in what immediately follows we shall use $S_{2}$ for four $t$ 's as such.

From the relations for four things, viz.,

$$
t^{4}-S_{1} t^{3}+S_{2} t^{2}-S_{3} t+S_{1}=0,
$$


we get

$$
\begin{gathered}
\left(t^{4}+S_{2} t^{2}+S_{4}\right)^{2}=\left(S_{1} t^{3}+S_{3} t\right)^{2}, \\
t^{8}-\sigma_{1} t^{6}+\sigma_{2} t^{4}-\sigma_{3} t^{2}+\sigma_{4}=0, \\
\sigma_{1}=S_{1}{ }^{2}-2 S_{2}, \quad \sigma_{2}=S_{2}{ }^{2}-2 S_{1} S_{3}+2 S_{4}, \\
\sigma_{3}=S_{3}{ }^{2}-2 S_{2} S_{4}, \quad \sigma_{4}=S_{4}{ }^{2} .
\end{gathered}
$$

Here the $\sigma$ 's are symmetric functions of $t_{i}^{2}(i=1,2,3,4)$ and therefore unaffected by a change of sign of $t_{i}$; but it is simply by this change of sign of $t_{i}$ in the equation of the original circle that we obtain those for the new circles. Hence, if in the equation for the center of the original circle,

$$
A B x=1+S_{2}+S_{4}-S_{1}\left(S_{1}+S_{3}\right)
$$

we substitute $-\sigma_{1}-2 S_{2}$ for $-S_{1}^{2}$ and $\frac{1}{2} \sigma_{2}^{2}-\frac{1}{2} S_{2}^{2}-S_{4}$ for $-S_{1} S_{3}$, it becomes

$$
A B x=1-\sigma_{1}+\frac{1}{2} \sigma_{2}-S_{2}-\frac{1}{2} S_{2}^{2},
$$

in which $A B$, the $\sigma$ 's, and $S_{4}$ are constants in so far as a change of sign of one $t$, or of two at a time, is concerned. The $S_{2}$ 's of course vary, but $S_{2}$ may be used as a parameter; and for the proper values of $S_{2}$, the equation picks up the eight centers of the second circles of the 4-line.

It is natural to divide the eight second circles into two sets-one for a reversal of one line, and another for a reversal of two. To this latter set belongs the original circle. Now $\bar{S}_{2}=S_{2} / S_{4}$. Let $S_{2}$ vary and call it $x$. Then

$$
S_{4} x-x=0
$$

the line of centers of the elliptic set of center circles. $S_{2}$, then, for a change of sign, runs around a line as $t$ runs around the unit circle. For a change of one line, we have $S_{2}$ tracing out the line

for a change of two lines,

$$
S_{4} x+\bar{x}=0
$$

$$
S_{4} x-\bar{x}=0
$$

As $S_{2}$ traces the latter line, (36) picks up the centers of four second circles; as it traces the former, the other four are picked up. What curve is represented by (36) as $S_{2}$ varies? Let $S_{4}$ be positive. If, then, $S_{2}$ is eliminated by means of (30) and its conjugate, we have

$$
(x-\bar{x})^{2}+\frac{2\left[S_{4}\left(S_{4}-1\right)-\left(1-\sigma_{1}+\sigma_{3}-\sigma_{4}\right)\right]^{\prime} x}{A B}
$$




$$
\begin{aligned}
& -\frac{2\left[\left(S_{4}-1\right)-\left(1-\sigma_{1}+\sigma_{3}-\sigma_{4}\right)\right] \bar{x}}{A B} \\
& -\frac{2\left(S_{4}-1\right)\left[S_{4}\left(1-\sigma_{1}+\frac{1}{2} \sigma_{2}\right)-\left(\sigma_{4}-\sigma_{3}+\frac{1}{2} \sigma_{2}\right)\right]}{A^{2} B^{2}} \\
& +\frac{\left(1-\sigma_{1}+\sigma_{3}-\sigma_{4}\right)^{2}}{A^{2} B^{2}}=0 .
\end{aligned}
$$

This is the equation of a parabola. Thus, the simplest curve connected with the four centers of the original second circle and the three corresponding to a reversal of two lines is the particular parabola (31).

Likewise, the particular parabola on the four centers of the second circles corresponding to a reversal of one line is

$$
\begin{aligned}
(x-\bar{x})^{2} & +\frac{2\left[S_{4}\left(S_{4}+1\right)-\left(1-\sigma_{1}+\sigma_{3}-\sigma_{4}\right)\right] x}{A B} \\
& +\frac{2\left[\left(S_{4}+1\right)+\left(1-\sigma_{1}+\sigma_{3}-\sigma_{4}\right)\right] \bar{x}}{A B} \\
& -\frac{2\left(S_{4}+1\right)\left[S_{4}\left(1-\sigma_{1}+\frac{1}{2} \sigma_{2}\right)+\sigma_{4}-\sigma_{3}+\frac{1}{2} \sigma_{2}\right]}{A^{2} B^{2}} \\
& +\frac{\left(1-\sigma_{1}+\sigma_{3}-\sigma_{4}\right)^{2}}{A^{2} B^{2}}=0 .
\end{aligned}
$$

When (31) is arranged as to $\bar{x}$, it becomes

$$
\begin{aligned}
\bar{x}^{2}- & \left\{2 x+\frac{\left[2\left(S_{4}-1\right)-2\left(1-\sigma_{1}+\sigma_{3}-\sigma_{4}\right)\right]}{A B}\right\} \bar{x}+x^{2} \\
& +\left\{\frac{2 S_{4}\left(S_{4}-1\right)-2\left(1-\sigma_{1}+\sigma_{3}-\sigma_{4}\right)}{A B}\right\} x \\
& -\frac{2\left(S_{4}-1\right)\left[S_{4}\left(1-\sigma_{1}+\frac{1}{2} \sigma_{2}\right)-\left(\sigma_{4}-\sigma_{3}+\frac{1}{2} \sigma_{2}\right)\right]}{A^{2} B^{2}} \\
& +\frac{\left(1-\sigma_{1}+\sigma_{3}-\sigma_{4}\right)^{2}}{A^{2} B^{2}}=0 .
\end{aligned}
$$

If the discriminant of this equation be set equal to zero, we obtain the focus. One root of the discriminant equation is infinite. The other is given by

$$
\begin{aligned}
\frac{2\left(S_{4}-1\right)^{2}}{A B} x & =\frac{\left(S_{4}-1\right)^{2}}{A^{2} B^{2}}-\frac{2\left(S_{4}-1\right)\left(1-\sigma_{1}+\sigma_{3}-\sigma_{4}\right)}{A^{2} B^{2}} \\
& +\frac{2 S_{4}\left(S_{4}-1\right)\left(1-\sigma_{1}+\frac{1}{2} \sigma_{2}\right)}{A^{2} B^{2}}-\frac{2\left(S_{4}-1\right)\left(\sigma_{4}-\sigma_{3}+\frac{1}{2} \sigma_{2}\right)}{A^{2} B^{2}},
\end{aligned}
$$


so that

$$
x=\frac{1+2\left(1-\sigma_{1}+\frac{1}{2} \sigma_{2}\right)}{2 A B}
$$

gives the focus. The focus of the parabola for $S_{4}$ negative is also given by $(F)$. Hence,

The two parabolas on which are the centers of the eight second circles of a 4-line arc confocal and hence intersect each other orthogonally.

Substitution shows that these two parabolas are on the points

$$
x=\frac{1-\sigma_{1}+\frac{1}{2} \sigma_{2}}{A B} \quad x=\frac{1-\sigma_{1}+\frac{1}{2} \sigma_{2}}{A B}-\frac{S_{4}^{2}-1}{2 A B} .
$$

The line on these intersections is $x+\bar{x}=1$. Whence,

Given a parabola; the two parabolas (31) and (32) corresponding to any four directed lines of the given parabola always intersect on its directrix orthogonally.

Further, the polars of the focus $(F)$ with reference to (37) and (38) are, respectively,

$$
x+\bar{x}=1+\frac{S_{4}}{A B}, \quad x+x=1-\frac{S_{4}}{A B},
$$

while their common axis is

$$
x-\bar{x}=\frac{1-\sigma_{1}+\sigma_{3}-\sigma_{4}}{A B}+\frac{1-\sigma_{4}}{2 A B} .
$$

Their focus, however, does not satisfy the equation of the directrix, $x+\bar{x}=1$, of the original parabola. Hence,

The parabolas (31) and (32) are not of the same size, but are similar, and similarly placed, ${ }^{*}$ to the original parabola.

The tangents at the point

$$
x=\frac{1-\sigma_{1}+\frac{1}{2} \sigma_{2}}{A B} \equiv c
$$

to (31) and (32) are, respectively,

$$
S_{4} x-\bar{x}-S_{4} c+\bar{c}=0, \quad S_{4} x+\bar{x}-S_{4} c-\bar{c}=0 .
$$

These tangents are important in the discussion that follows.

Consider next the equation of the second circle of the directed 4-line, expressed parametrically in terms of $S_{2}$, and $S_{4}$ positive:

$$
A B x-1+\sigma_{1}-\frac{1}{2} \sigma_{2}+S_{2}+\frac{1}{2} S_{2}^{2}=\frac{S_{2}}{2 t}\left(S_{1}+S_{3}\right) .
$$

The conjugate is

$$
A B x-\sigma_{4}+\sigma_{3}-\frac{1}{2} \sigma_{2}+S_{2} S_{4}+\frac{1}{2} S_{2}^{2}=\frac{S_{2} t}{2}\left(S_{1}+S_{3}\right) .
$$

\footnotetext{
* Salmon, Conic Sections, p. 223.
} 
Multiply these equations member by member, and, in the product, put $\sigma_{1}+2 S_{2}$ for $S_{1}^{2}, \quad-\sigma_{2}+S_{2}^{2}+2 S_{4}$ for $2 S_{1} S_{3}$, and $\sigma_{3}+2 S_{2} S_{4}$ for $S_{3}^{2}$. Employ the former abbreviation $c$ and note that $\bar{c}=\left(\sigma_{4}-\sigma_{3}+\frac{1}{2} \sigma_{2}\right) / A B$. Also set, $S_{4}$ being positive,

We obtain

$$
k=\left(1-\frac{1}{2} \sigma_{1}+\frac{1}{2} \sigma_{3}-S_{4}\right) / A B .
$$

$$
\begin{aligned}
2 A B(x \bar{x}-\bar{c} x-c \bar{x}+c \bar{c})+2 S_{2}\left(S_{4} x+\bar{x}-S_{4} c-\bar{c}\right) & \\
& +S_{2}^{2}(x+\bar{x}-c-k)=0 .
\end{aligned}
$$

This is the equation of the second circle expressed parametrically, $S_{2}$ being the parameter. $c, \bar{c}$, and $A B$ are constants with respect to a change of sign of the parameters, or $t^{\prime}$ 's of the four lines. $S_{4}$ is constant for a change of sign of one, or of two, $t$ 's. It is evident that (33) is self-conjugate as a whole, while each of its parentheses equated to zero is also self-conjugate, $c+k$ being real. Moreover, the part included in the first parenthesis is a point circle; those in the second and third parentheses are lines, which, however, in our inversive geometry may be considered as circles. Thus, (33) is of the form

$$
\lambda^{2} U+2 \lambda V+W=0,
$$

where $U=0, V=0$, and $W=0$ are circles. But (34) represents a quadratic system of circles.* Therefore,

The second circles of a directed 4-line belong to two quadratic systems of circles; four to the system for which $S_{4}$ is positive, four to that for which it is negative, and this in contrast to the linear systems of center, or first, circles for the same lines.

Since the lines (circles)

$$
S_{4} x-\bar{x}-S_{4} c+\bar{c}=0, \quad S_{4} x+\bar{x}-S_{4} c-\bar{c}=0
$$

belong each to a quadratic system of circles, in fact are themselves base circles of their respective systems, and since the equation of the point circle

$$
x \bar{x}-\bar{c} x-c \bar{x}+c \bar{c}=0
$$

does not contain $S_{4}$, we have the theorem:

The tangents at the point $c$ to the parabolas (31) and (32) are base circles each of a quadratic system. Also the point circle is common to the two systems, while the base lines (circles) $x+\bar{x}-c-k=0$ are parallel to the directrices of (31) and (32) as well as to that of the original parabola.

In the equation of a circle in conjugate coördinates

$$
x \bar{x}-a \bar{x}-\bar{a} x+a \bar{a}-b \bar{b}=0
$$

* See Salmon, Higher Plane Curves, French Edition, p. 343. 
the center is $a$. In the quadratic system of circles just derived, viz., $2 A B(x-c)(\bar{x}-\bar{c})+2 S_{2}\left(S_{4} x+\bar{x}-S_{4} c-\bar{c}\right)+S_{2}^{2}(x+\bar{x}-\bar{c}-k)=0$, given an $S_{2}$, we have a circle; but if we are given an $x_{1}$ there are two values of $S_{2}$, that is, there are two circles on a point. Further, given an $S_{2}$, the center of the resulting circle is the negative of the coefficient of $\bar{x}$, which in this case is

$$
l=\left(1-\sigma_{1}+\frac{1}{2} \sigma_{2}-S_{2}-\frac{1}{2} S_{2}^{2}\right) / A B .
$$

But $x=l$ was the parabola which contained the centers of four circles of one set of the two into which the second circles of the 4-line are divided. Hence

The center of every circle belonging to a quadratic system arising from a directed 4-line is on a parabola; (31) contains the centers of the one system, (32) those of the other.

Again, the three base circles of the quadratic system for which $S_{4}$ is positive are

$$
\begin{aligned}
& W \equiv x \bar{x}-c \bar{x}-\bar{c} x+c \bar{c}=0, \\
& V \equiv S_{4} x+\bar{x}-S_{4} c-\bar{c}=0, \\
& U \equiv x+\bar{x}-\bar{c}-k=0 .
\end{aligned}
$$

A circle orthogonal to these circles must have its center at the intersection of $U$ and $V$ and must be on the point $c$. The intersector. of $U$ and $V$ is

Hence the orthogonal circle is

$$
\frac{S_{4} c-k}{S_{4}-1}
$$

$$
x \bar{x}-\frac{k-S_{4} c}{1-S_{4}} \bar{x}-\frac{\bar{c}-S_{4} \bar{k}}{1-S_{4}} x+\frac{\bar{c} k-S_{4} c k}{1-S_{4}}=0 .
$$

This circle is orthogonal to every circle of the system, for the Jacobian of $W, V$, and $U$ (the point $c$ being the origin), is

$$
\left|\begin{array}{ccc}
\bar{x} & S_{4} & 1 \\
x & 1 & 1 \\
0 & S_{4} x+\bar{x} & x+\bar{x}+2(c-k)
\end{array}\right|=0,
$$

which is the same as (35).

The tangent at the point $c$, of (35) is

$$
S_{4} x-\bar{x}-S_{4} c+\bar{c}=0 .
$$

But this is also the tangent at the same point of the parabola (31). We see, therefore, that 
The parabola (31), which may be called with (32) a focal parabola,* and the Jacobian circle of the corresponding quadratic system have contact at the point $c$.

Also, since there are two quadratic systems and therefore two Jacobian circles, the two Jacobian circles of the quadratic systems arising from a directed 4-line intersect orthogonally.

Two of the intersections of the Jacobian circle for $S_{4}$ positive and the focal parabola (31) are at $c$, as we have just seen. If $c$ is the origin, the other two intersections are

$$
x=-\frac{1}{2}\left(1-\frac{1-S_{4}}{A B}\right) \pm \frac{S_{4}}{\sqrt{A B}} .
$$

Bicircular quartics $\dagger$ may be regarded as the envelope of the quadratic system of circles

$$
W+2 \lambda V+\lambda^{2} U=0 .
$$

Again, all circles of the system

$$
\alpha U+\beta V+\gamma W=0
$$

have a common orthogonal circle. Hence those of the former system have such a circle, and their centers are on a conic. Therefore a bicircular quartic may be considered as the envelope of a circle whose center moves on a fixed conic $F$, say, and which cuts a fixed circle orthogonally. However, if the conic $F$ touch the line joining the two common points of the circles of the system, the quartic degenerates into a circular cubic and the line at infinity.

Now, this last statement characterizes precisely the condition of affairs in the present case, since, as has been shown, the systems of circles under consideration contain only those with centers on parabolas, and each system has a common orthogonal (or Jacobian) circle, and is itself of quadratic form. Therefore

The envelope of the quadratic systems of circles belonging to a directed 4-line is a circular cubic.

If we eliminate the parameter $S_{2}$ from (39) we have, for $S_{4}$ positive,

$$
2 A B[(x-c)(\bar{x}-\bar{c})][x+\bar{x}-(\bar{c}+k)]=\left(S_{4} x+\bar{x}-S_{4} c-\bar{c}\right)^{2},
$$

the equation of the circular cubic. Since this may be written in the form $W U I=V^{2} I^{2}$, or $W U=V^{2} I, I$ being the line at infinity, it is obvious that $U$ touches the cubic where $U$ meets $V$ and intersects the cubic where $V$ intersects $I$. Also $W$ touches the cubic where $W$ meets $V$ and intersects the cubic at the circular points. Moreover as $U$ meets the cubic at infinity, $U$ is parallel to an asymptote.

* Salmon, Higher Plane Curves, French edition, p. 346; Basset, Cubic and Quartic Curves, p. 139; Casey, Transactions of the Royal Irish Academy, vol. 24.

† See Salmon, Higher Plane Curves, French Edition, p. 243 et seq. 
When the Jacobian circle has contact with the focal parabola, the point of contact is a double point on the envelope of the system of circles. Hence

The circular cubic which is the envelope of the quadratic system of circles of a directed 4-line is rational.

The double point is the point $c$. If this point is taken as origin, the cubic (37), for $S_{4}$ positive, becomes

$$
x \bar{x}(x+\bar{x})+\left(\frac{1}{2}-\frac{1+S_{4}^{2}}{2 A B}\right) x \bar{x}-\frac{S_{4}^{2}}{2 A B} x^{2}-\frac{1}{2 A B} \bar{x}^{2}=0 .
$$

It is easy to verify that the tangent at the origin proves illusory. Further, since (44) does not contain $S_{4}$ in the first power, we may add:

The two quadratic systems of circles of a directed 4-line have the same circular cubic as envelope.

Four of the foci of the cubic are the four intersections of the focal parabola (31) and the corresponding Jacobian circle. As two of these intersections coincide at $c$, this point is a double focus, while the other two are

$$
x=-\frac{1}{2}\left(1-\frac{1-S_{4}^{2}}{A B}\right) \pm \frac{S_{4}}{\sqrt{A B}} .
$$

That these are the foci is readily verified by equating to zero the discriminant as to $\bar{x}$ of equation (38). Using the focal parabola (38) and the corresponding Jacobian circle, the point $c$ is again a double focus, while the two corresponding to (39) are imaginary.

We have seen that $U$ is parallel to an asymptote of the cubic. In Cartesian coordinates with the focus of the original parabola at the origin, (38) becomes

$$
2 x^{3}+2 x y^{2}+\left(\frac{1}{2}-\frac{1+S_{4}^{2}}{A B}\right) x^{2}+\frac{1}{2} y^{2}+\left(\frac{1-S_{4}^{2}}{A B}\right) i x y=0 \text {. }
$$

The coefficient of $y^{2}$ vanishes for $x=1 / 4$. Hence

The envelope circular cubic derived from any four directed lines of a given parabola has the tangent at the vertex of the parabola as asymptote.

If in (38) we write $t x=\bar{x}$, we obtain the parametric equations of the cubic:

where

$$
x=\frac{\bar{b} t^{2}-a t+b}{t(t+1)}, \quad \bar{x}=\frac{b t^{2}-a t+b}{t+1},
$$

$$
a=\frac{1}{2}-\frac{1+S_{4}^{2}}{2 A B}, \quad b=\frac{S_{4}^{2}}{2 A B}
$$

Thus the fundamental involution is given by

$$
(a+\bar{b}) \bar{b} S_{3}-b \bar{b} S_{2}-b \bar{b} S_{1}+(a+b) b=0 .
$$


The cubic giving the points of inflexion is

$$
t^{3}-\frac{3 b}{a+\bar{b}} t^{2}-\frac{3 b}{a+\bar{b}} t+\frac{(a+b) b}{(a+\bar{b}) \bar{b}}=0 .
$$

The line on the points of inflexion is

$$
(a+b) x+(a+\bar{b}) \bar{x}+a^{2}-4 \bar{b} \bar{b}=0 .
$$

It is easily shown that the cubic (38) is also the envelope of the system of circles on the origin whose centers $\alpha$ satisfy the equation of the parabola

$$
\begin{aligned}
(\alpha-\bar{\alpha})^{2}+\left(1-\frac{1-S_{4}^{2}}{A B}\right) \alpha+( & \left.+\frac{1-S_{4}^{2}}{A B}\right) \bar{\alpha} \\
+ & {\left[\frac{1}{2}-\frac{\left(1-S_{4}^{2}\right)}{2 A B}\right]\left[\frac{1}{2}-\frac{\left(1+S_{4}\right)^{2}}{2 A B}\right]=0 . }
\end{aligned}
$$

\$4. On center circles for a directed 5-line.

Three directed lines have an equidistant point, depending on the assigned directions of the lines. Four such lines have a circle on their four equidistant points, one for each three of the four lines. This is the center circle for four lines. We have seen that by reversing the direction of the lines, we get eight center circles for the four lines. Consider five lines. For every four there is a center circle, and hence five such circles in a 5-line. The five centers of these circles are on a circle themselves. This circle is the "first " or "center circle" * of the 5-line. However, if one or more lines are reversed in directions we get a different center circle. In fact, if we reverse one line at a time, we get five new center circles; if we reverse two lines, we get ten new center circles. Thus, in a directed 5-line there are sixteen center circles in all.

It has been observed that the eight centers of the circles for a 4-line lie on two perpendicular lines. How do the centers for a 5 -line lie?

First, put a condition on the 5 lines, and let them be lines of a parabola. Then the lines are of form

$$
t x-\frac{\bar{x}}{t}=\frac{t}{1-t^{2}}
$$

whence the center circle for four lines is

and that for five is

$$
2 x=\frac{1}{A}+\frac{1}{B}-t\left(\frac{1}{A}-\frac{1}{B}\right) \quad(A, B \text { for four lines }),
$$

$$
2 x=\frac{1}{A}+\frac{1}{B}-t\left(\frac{1}{A}-\frac{1}{B}\right) \quad(A, B \text { for five lines ), }
$$

* Loud, loc. cit., p. 325, Theorem I. 
or in conjugate coördinates

$$
2 x \bar{x}-\left(\frac{1}{A}+\frac{1}{B}\right) \bar{x}+S_{5}\left(\frac{1}{A}-\frac{1}{B}\right) x=0 .
$$

This circle is on the origin and the form is similar for any odd number of lines. Hence,

If $N$ is odd, the $2^{n-1}$ center circles for $n$ directed lines of a parabola are on the origin.

The center of the center circle for five lines is

Thus

$$
2 x=\frac{1}{A}+\frac{1}{B} \text {. }
$$

$$
2 A B x=B+A, \quad-2 A B \bar{x}=S_{5}(B-A) .
$$

Consequently we have

$$
A B\left(S_{5} x-\bar{x}\right)=S_{5} B, \quad A B\left(S_{5} x+x\right)=S_{5} A,
$$

whence finally

$$
S_{5}^{2} x^{2}-\bar{x}^{2}=\frac{S_{5}^{2}}{A B}
$$

This is the equation of a rectangular hyperbola, and is the curve on which are the centers of the sixteen center circles for the five lines. This is so, because (45) is not affected by the change of sign of any number of the $t$ 's, i. e., parameters of the lines. Evidently the result is similar for any odd number of lines. Hence again

If $N$ is odd, the $2^{n-1}$ centers of the center circles for $n$ directed lines of a parabola are on a rectangular hyperbola.

For any odd number of lines the center circle was

$$
x \bar{x}-\alpha \bar{x}-\bar{\alpha} x=0
$$

$$
\left[\alpha=\frac{1}{2}\left(\frac{1}{A}+\frac{1}{B}\right)\right]
$$

The center $\alpha$ satisfies the equation

$$
S_{n}^{2} x^{2}-\bar{x}^{2}=\frac{S_{n}^{2}}{A B}
$$

Considering $\alpha$ and $\bar{\alpha}$ as parameters connected by the equation just written, we obtain

$$
S_{n}^{2} \alpha x+\bar{\alpha} \bar{x}=0 .
$$

This, in connection with $\left(44^{\prime}\right)$, gives

$$
A B x^{2} \bar{x}^{2}=\bar{x}^{2}-S_{n}^{2} x^{2},
$$

which is a lemniscate. Therefore, 
The envelope of the system of circles to which belong the center circles arising from any odd number of directed lines of a parabola is a lemniscate.

It is evident from the equations that the asymptotes of the rectangular hyperbola are the nodal tangents of the corresponding lemniscate.

For the purpose of handling five directed lines free of any condition on them, it is often desirable to take five tangents of the so-called double parabola.* This curve is a class cubic with the line at infinity a double line. This accounts for three constants, so that six remain. Hence six lines uniquely determine this parabola, of which a directed tangent is

$$
t x-\frac{\bar{x}}{t}=\frac{c_{1} t}{\lambda_{1}^{2}\left(\lambda_{1}^{2}-t^{2}\right)}+\frac{c_{2} t}{\lambda_{2}^{2}\left(\lambda_{2}^{2}-t^{2}\right)},
$$

where $c_{i}$ is an arbitrary constant and $\lambda_{i}$ an arbitrary turn. Also, $\bar{c}_{i}=c_{i} / \lambda_{i}^{6}$. The center circle for five lines now is

$$
\begin{gathered}
2 x=\sum^{2} \frac{c_{1}}{\lambda_{1}}\left(\frac{\lambda_{1}-t}{P_{1}}+\frac{\lambda_{1}+t}{Q_{1}}\right), \\
P_{1} \equiv\left(\lambda_{1}-t_{1}\right)\left(\lambda_{1}-t_{2}\right)\left(\lambda_{1}-t_{3}\right)\left(\lambda_{1}-t_{4}\right)\left(\lambda_{1}-t_{5}\right), \\
Q_{1} \equiv\left(\lambda_{1}+t_{1}\right)\left(\lambda_{1}+t_{2}\right)\left(\lambda_{1}+t_{3}\right)\left(\lambda_{1}+t_{4}\right)\left(\lambda_{1}+t_{5}\right) .
\end{gathered}
$$

The center of the circle is therefore given by

$$
2 x=c_{1}\left(\frac{1}{P_{1}}+\frac{1}{Q_{1}}\right)+c_{2}\left(\frac{1}{P_{2}}+\frac{1}{Q_{2}}\right) .
$$

There are fifteen similar expressions for the centers of the circles obtained on reversing one or more lines of the five, namely, by changing the signs of the corresponding $t$ 's.

Equation (48) may be written in the form

$$
\frac{x-M_{5}}{M_{1}}=\frac{M_{3}}{M_{1}} S_{2}+S_{4}, \quad M_{j} \equiv \frac{c_{1} \lambda_{1}^{j}}{P_{1} Q_{1}}+\frac{x_{2} \lambda_{2}^{j}}{P_{2} Q_{2}} .
$$

Since the conjugate of $M_{3} / M_{1}$ equals its reciprocal, it is a turn; call it $\mu^{2}$. The sum of the two expressions

$$
\mu^{5} \pm \mu^{4} S_{1}+\mu^{3} S_{2} \pm \mu^{2} S_{3}+\mu S_{4} \pm S_{6}
$$

is $2 \mu^{5}+2 \mu r$, where $r=\mu^{2} S_{2}+S_{4}$. From the resulting value of $r$, we get

Also

$$
\frac{2 \mu\left(x-M_{5}\right)}{M_{1}}+2 \mu^{5}=\prod_{i=1}^{5}\left(\mu+t_{i}\right)+\prod_{i=1}^{5}\left(\mu-t_{i}\right) .
$$

$$
\frac{2 \mu^{4}\left(\bar{x}-M_{5}\right)}{-M_{3}}+2 S_{5}^{2}=S_{5} \Pi\left(\mu+t_{i}\right)-S_{5} \Pi\left(\mu-t_{i}\right) .
$$

*W. K. Currord, Mathematical Papers, p. 46.

Trans. Am. Math. Soc. 18 
Adding and subtracting these last two equations and multiplying the results, we have

$$
\mu^{2} S_{5}^{2}\left[\frac{x-M_{5}}{M_{1}}+\mu^{4}\right]^{2}-\left[\frac{\left(x-M_{5}\right)}{-M_{3}}+S_{5}^{2}\right]^{2}=S_{5}^{2} \prod_{i=1}^{5}\left(\mu^{2}-t_{i}^{2}\right),
$$

an equation unaffected by the change of sign of one or more parameters of the lines. Therefore it represents the curve on which are the different centers of the center circles for the given five lines. It is a rectangular hyperbola. Hence

The centers of the sixteen center-circles arising from any five directed lines are on a rectangular hyperbola.

\section{§5. On center circles for a directed 6-line.}

Since, as we have seen, six lines uniquely determine a double parabola, we may consider six lines of this curve as a general six-line. It is convenient, then, in discussing the center circles for six lines to take theses as tangents of a double parabola.

By reversing one line at a time we obtain six new center circles of the 6-line. By reversing two lines simultaneously, there arise as many new circles as there are ways of selecting two things from six, viz., fifteen. By a reversal of the direction of three lines ten new circles are produced. For, three things can be selected from six in twenty ways, but a simultaneous reversal of lines 1, 2, 3 , is, for our problem, equivalent to a simultaneous reversal of 4,5 , and 6 . Hence the number is one-half of twenty, or ten. Thus in all there are 32 circles for the 6 -line.

The equation of the center circle for six directed lines of a double parabola is

$$
2 x=\sum^{2} \frac{c_{1}}{\lambda_{1}}\left(\frac{\lambda_{1}-t}{P_{1}}+\frac{\lambda_{1}+t}{Q_{1}}\right),
$$

where now $P_{i}$ and $Q_{i}$ are formed for six factors. The center of the circle is

whence

$$
2 x=c_{1}\left(\frac{1}{P_{1}}+\frac{1}{Q_{1}}\right)+c_{2}\left(\frac{1}{P_{2}}+\frac{1}{Q_{2}}\right),
$$

$$
2 \bar{x}=c_{1} S_{6}\left(\frac{1}{P_{1}}+\frac{1}{Q_{1}}\right)+c_{2} S_{6}\left(\frac{1}{P_{2}}+\frac{1}{Q_{2}}\right) .
$$

Subtracting, we have

$$
S_{6} x-\bar{x}=0 .
$$

Hence the center of the center circle for six lines is on this line which is itself on the origin. Now $S_{6}$ is positive for the original circle and for those arising 
from a reversal of two lines. Thus sixteen centers are on this line. Since $S_{6}$ becomes negative for a change of sign of one $t \mathrm{nr}$ of three $t$ 's, sixteen other centers are on the line

$$
S_{6} x+\bar{x}=0,
$$

a perpendicular to the first line and also on the origin. Therefore The 32 centers of the center circles of a directed 6-line are on two perpendicular lines, 16 on each line.

JoHNS HopkINS UNIVERSITY, March, 1910. 